\title{
Imagined lines fail to induce contour masking*
}

\author{
RAY OVER† and JACK BROERSE \\ University of Queensland, St. Lucia, Australia 4067
}

Twelve Ss proficient in visual imagery were tested in a study to compare contour masking by real and imaginary lines. Detection of a vertical line was impaired following inspection of a vertical grating but was unaffected by exposure to a horizontal grating. When Ss were required to imagine a dot pattern as either a vertical or horizontal grating prior to judging whether a vertical line was present or absent in the display, the orientation that $\mathrm{S}$ was instructed to imagine did not influence detection of the target. This evidence that specific visual imagery does not mask a dimensionally similar visual target is contrasted with other data indicating that isomodal masking occurs when complex visual and auditory imagery and target stimuli are used.

Segal \& Fusella (1970) examined sensitivity for auditory and visual signals in a task in which Ss were required to imagine pictures and sounds when judging whether the target stimulus was present or absent. Detectability ( $d^{\prime}$ ) was more impaired when the image and signal were both auditory or both visual than with crossmodal arrangements. Segal and Fusella proposed that imagery raised the activity level in specific sensory pathways and imnaired detection by functioning as an internal signal that was confused with the external signal. However, in Segal and Fusella's experiment, both the target and imagined stimuli were dimensionally complex. The visual target was a blue arrow and the auditory target a harmonica chord. Prior to presentation of the target, $S$ was required to imagine such conditions as a table, a tree, a dinosaur (visual), and a phone ringing, a dog barking, an oboe playing (auditory).

The present experiment considers whether highly specific visual imagery masks a dimensionally simple visual target. It is asked whether imagined lines can impair the detection of real lines as a function of the difference in orientation between the real and imagined lines. Masking studies with real lines (e.g., Campbell \& Kulikowski, 1966; Houlihan \& Sekuler, 1968) have shown that the detectability of a target line shown in the same part of the visual field as an adaptation grating is most impaired when the line and grating are identical

*This research was supported by a grant to the first author from the Australian Research Grants Committee. The authors are grateful to Ann-Marie parker for her assistance in selection of $S s$ and for conducting the experiment.

tRequests for reprints should be sent to Ray Over, Department of Psychology. University of Queensland, St. Lucia, A ustralia 4067 in orientation, and improves as their tilts differ. In particular, detection of a vertical target line is impaired following inspection of a vertical grating but is unaffected by exposure to a horizontal grating (Over, Broerse, \& Crassini, 1972). The issue of interest in the present experiment was whether instructions to imagine a symmetrical dot pattern as either a vertical or a horizontal grating would differentially influence detection of a vertical grating presented in the same part of the visual field as the dot pattern.

\section{METHOD}

A forward masking paradigm was used, with stimuli displayed in separate channels of a Gerbrands tachistoscope (Model T-3B-1). On each trial, the $S$ viewed a fixation point for $1 \mathrm{sec}$, the inducing field for $300 \mathrm{msec}$, and the target (or a homogeneous test field on "catch" trials) for $10 \mathrm{msec}$. There was a dark interval of $120 \mathrm{msec}$ between offset of the fixation field and onset of the inducing field and of $10 \mathrm{msec}$ between offset of the inducing field and onset of the target. The S's task on each trial was to detect whether the target line, which subtended $1 \mathrm{deg} 42 \mathrm{~min} \times 6 \mathrm{~min}$, or the homogeneous test field had been presented after display of the inducing stimulus. Measures were obtained under three inducing conditions (real lines, imagined lines, blank field). With real lines, $S$ viewed a 5-deg 22-min square grating of 6-min-wide lines separated by $26 \mathrm{~min}$. The grating was formed by fixing matte white tape to black cardboard. Under imagery conditions, the inducing stimulus comprised 81 dots, each subtending $6 \mathrm{~min}$ in diam and separated center to center by $26 \mathrm{~min}$. The dots were arranged as a 9 by 9 square such that, had a horizontal and a vertical grating been superimposed, a dot would have appeared at every intersection of the resulting grid pattern. A homogeneous field equated in space-average luminance $\left(1.72 \mathrm{~cd} / \mathrm{m}^{2}\right)$ to the grating and dot display served as the third inducing condition. The fixation point coincided spatially with the centers of the inducing figure and target line and was presented on a blank field maintained at $14 \mathrm{~cd} / \mathrm{m}^{2}$

Twelve Ss were selected from a large group of undergraduate students in terms of scores on the shortened version of the Betts QMI Vividness of Imagery Scale (Sheehan, 1967). The mean imagery rating of the visual items in the scale by the Ss was 2.03 $(\mathrm{SD}=.87)$, compared with $2.88(\mathrm{SD}=$ 1.32 ) for the group as a whole.

Nine detection measures (one at each of three target orientations with the grating, the dot pattern, and the blank field as inducing stimuli) were obtained from each $S$ in each of two sessions. All Ss made judgments with the blank field as the inducing stimulus. Half the group was tested with vertical and the other half with horizontal, real, and imagined lines as inducing stimuli. In each case, perception was measured with the target line displayed 0,15 , and $30 \mathrm{deg}$ from vertical. Detection of the target was determined by a blockwise tracking procedure (Houlihan \& Sekuler, 1968). By this method, the threshold for detection under a specific stimulus condition was taken as the Iuminance value of the target needed for S to identify correctly $75 \%$ of six target and six "catch" trials presented in random order. The target was initially displayed at a luminance level at which, during preliminary testing with the homogeneous inducing field, the $S$ had performed at chance level. The luminance of the target was increased in $1.72-\mathrm{cd} / \mathrm{m}^{2}$ steps over successive blocks until an accuracy level of $75 \%$ or greater was attained over a block. If $\mathrm{S}$ made nine or more correct responses on the initial block, the luminance of the target was decreased in $1.72-\mathrm{cd} / \mathrm{m}^{2}$ steps. The order of testing the three inducing conditions and three orientations of the target line was varied across $S s$ and between sessions by three 9 by 9 Latin squares, with three sequences unused.

Testing $w$ as conducted in a darkened room, and each $\mathrm{S}$ received $10 \mathrm{~min}$ of dark adaptation prior to the start of testing. There was a $10-\mathrm{sec}$ dark interval between successive trials. On each trial, $S$ initiated the stimulus presentation at E's direction, and under imagery conditions, $\mathbf{S}$ was told to initiate the display only when he felt ready to imagine the dot pattern as lines. All Ss reported that they were readily and consistently able to imagine the dot pattern as a grating during the $300-\mathrm{msec}$ presentation interval. 


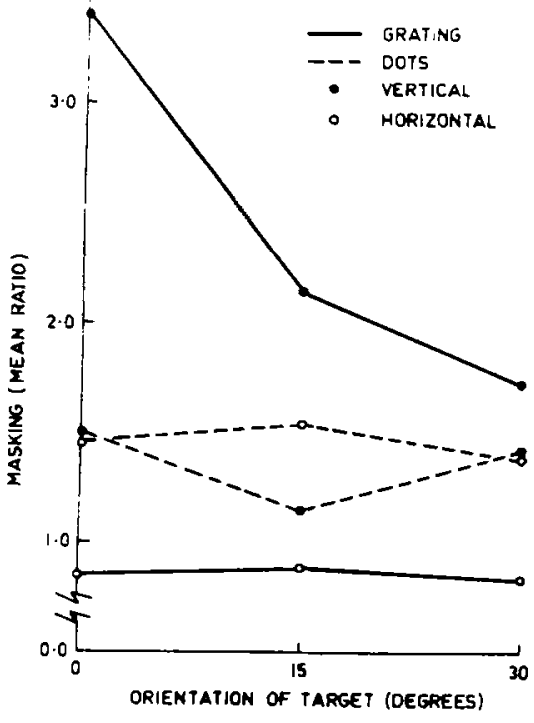

Fig. 1. Mean masking (impairment in detection of the target line) as a function of target orientation and the spatial value (real or imagined) of the inspection stimulus.

\section{RESULTS}

The detectability of the target line was measured as the luminance value at which $\mathrm{S}$ was able to differentiate target and "catch" trials with $75 \%$ accuracy. This value was determined by extrapolation when necessary. Luminance thresholds established at the different orientations of the target line with grating and dot inducing fields were converted to ratio values relative to measures found using the blank inducing field. The mean ratios determined in this manner are shown in Fig. 1. Masking (elevation in threshold following exposure to patterned stimuli) requires that the ratio exceeds 1.0 ; facilitation (better detection following exposure to the grating or dots than to the blank field) has occurred if the ratio is less than 1.0 .

The three main effects were significant by analysis of variance: (A) orientation of the target line $[\mathrm{F}(2,20)=25.73, \mathrm{p}<.01] ;$ (B) type of inducing figure (dots vs lines) $[\mathrm{F}(1,20)=5.17, \quad \mathrm{p}<.05]$; (C) orientation (vertical vs horizontal) of real or imagined lines $[F(1,20)=$ $19.00, p<.01]$. In addition, all interactions were significant: $A$ by $B$ $[F(2,20)=9.84, p<.01] ; A$ by $C$
$[F(2,20)=25.13, p<.01] ; B$ by $C$ $[F(1,10)=61.62, p<.01] ; A$ by $B$ by C $[F(2,20)=10.51, \quad p<.01]$. Comparisons based on Duncan's new multiple-range test indicated that the orientation at which $\mathrm{S}$ was instructed to imagine the dot pattern did not differentially modify detection as the orientation of the target line was changed. Nor did inspection of the grating of horizontal lines affect detection as a function of the orientation of the target. The difference in detection ratios between this latter condition and the imagery conditions probably arose because the inducing stimuli (lines vs dots) were equated in space-average rather than local luminance. When the inducing figure was the grating of vertical lines, detection improved progressively as the orientation of the target varied from vertical.

\section{DISCUSSION}

Exposure to a grating of real lines resulted in orientation-specific contour masking in a manner consistent with earlier studies (e.g., Campbell \& Kulikowski, 1966; Houlihan \& Sekuler, 1968; Over, Broerse, \& Crassini, 1972). In specific terms, the threshold for detection of a vertical target line was unaffected by prior display of a horizontal grating, but was impaired following presentation of a vertical grating. However, when the dot pattern was used as the inducing stimulus in place of the grating, instructions to imagine the array of dots as columns overtical lines or rows of horizontal lines did not selectively affect the detectability of the target line.

The present data, obtained within the masking context, are consistent with aftereffect measures reported by Singer \& Sheehan (1965). They found that a vertical line appears tilted in the counterclockwise direction when its orientation is judged following prolonged exposure to a clockwise tilted grating. It was not, however, possible to generate equivalent aftereffects by instructing Ss to imagine clockwise tilted lines prior to presentation of the vertical test line. This similarity between masking and aftereffect data is of interest in view of proposals (e.g., Over, 1971) that both effects occur through selective adaptation of spatially tuned detectors in the human visual system. The claim is that perception within each paradigm is distorted to the extent that the spatial properties of the test figure are normally signaled by detectors that are in an adapted state following inspection.

Segal \& Fusella (1970) demonstrated masking by imagery when multiattribute target and imaginary stimuli were used. Evidence that masking by imagery was greater within than between modalities led Segal and Fusella to suppose that the imagined stimulus resulted in sensory events that interacted with the neural signals generated by the target stimulus. The present study employed single-attribute imaginary and target stimuli and failed to provide evidence indicating that the imaginary stimulus affected neural processing of the actual stimulus. The discrepancy between the present results and Segal and Fusella's data may relate to the complexity of stimuli used in the two cases. It would be of interest to replicate Segal and Fusella's study employing a pure tone rather than a harmonica chord as the target stimulus and requiring $S s$ to imagine real tones rather than complex stimuli such as a phone ringing, a dog barking, and an oboe playing. If there is no masking by imagery with pure tones, it is probable that masking by complex imagery occurs through a feature-selective attentional mechanism rather than through direct neural interaction.

\section{REFERENCES}

CAMPBELL, $F, W_{. .}$\& KULIKOWSKI. J. J Orientational selectivity of the human visual system. Journal of Physiology. $1966,198,237-250$.

HOULIHAN, K.. \& SEKULER, R. W Contour interactions in visual masking. Journal of Experimental Psychology, $1968,77,281-285$

OVER, R. Comparison of normalization theory and neural enhancement explanation of negative aftereffects. Psychological Bulletin, 1971, 75. 225-243.

OVER, R., BROERSE, J., \& CRASSINI, B. Orientation illusion and masking in central and peripheral vision. Journal of Experimental Psychology, 1972, in press.

SEGAL, S. J., \& FUSELLA, V. Influence of imaged pictures and sounds on detection of visual and auditory signals. Journal of Experimental Psychology, 1970, 8, 458-464.

SHEEHAN, P. W. A shortened form of Betts' Questionnaire Upon Mental Imagery. Journal of Clinical Psychology, $1967,23,386-389$.

SINGER, G., \& SHEEHAN, P. W. The effects of demand characteristics on the figural after-effect with real and imaged inducing figures. American Joumal of Psychology, 1965, 78, 96-101. 